\title{
Net'O'Drom- \\ An Example for the Development of Networked Immersive VR Applications
}

\author{
Christoph Anthes ${ }^{1}$, Alexander Wilhelm² ${ }^{2}$, Roland Landertshamer ${ }^{1}$, \\ Helmut Bressler ${ }^{1}$, and Jens Volkert ${ }^{1}$ \\ ${ }^{1}$ GUP, Institute of Graphics and Parallel Processing \\ Johannes Kepler University, Altenbergerstraße 69, A-4040 Linz, Austria \\ canthes@gup . uni-linz.ac . at \\ 2 The Visioneers, \\ Fabrikstraße 2, A-4020 Linz, Austria \\ http://www. thevisioneers. com
}

\begin{abstract}
Developing Networked Virtual Environments (NVEs) is a challenging task. Designers and programmers of such an application can encounter many pitfalls. For a public event, a night of science, a networked Virtual Reality (VR) application had to be designed and implemented. This paper will describe the technical and conceptual design of this application - The Net'O'Drom game. Possible pitfalls during the design and implementation of NVEs will be pointed out and suggestions on how to overcome these issues will be given. A closer look at the problem areas of physics, network communication and graphical effects is taken.
\end{abstract}

\section{Introduction}

Networked graphics applications are becoming more and more common nowadays. If we take a closer look in the area of VR and NVEs, medical visualisations, safety training and architectural design are just a few application areas, where the users benefit from interaction in a networked environment. Not only the area of VR but also the area of multi-player games opens a vast market for networked graphics applications. The algorithms and distribution mechanisms between these two areas are very similar, but VR provides additional challenges like multi-wall displays, different interaction techniques and it has tighter constraints considering responsiveness.

This paper gives an overview on the design of a networked graphics application and takes a racing game as an example. The example of such a game seams to be ideal, since it consists of the common components usually needed in NVEs. The description of the components and the overall design of the Net'O'Drom game should give an idea on how to approach the development of an NVE. It should not be considred as a generic approach, but rather be an inspiring source for design ideas. Common issues which arise during the development of such a VE will be pointed out and suggestions on how to overcome these obstacles will be given. 
The following Section examines various design approaches in the field of VEs and NVEs. Afterwards the conceptual design from an artistic point of view will be given. Section 4 will describe the application design of the Net'O'Drom game. The next Chapters will focus on the developed physics engine, the network component and the effects used to enhance immersion and the gaming experience. The final Sections conclude the paper and provide an outlook into future developments.

\section{Related Work}

In general VR applications can be developed in many ways. Authoring systems allow experts of a given application domain, which have not much programming experience to design VEs by using graphical editors. Other systems like DIVE[1], ALICE[2] or AVANGO1 [3] rely on the use of scripting languages like TCL and Python. Through the use of graphical interfaces or scripting languages the design of a $\mathrm{VE}$ is more intuitive, but obvious drawbacks are limited flexibility and a higher need of computational power. Many domain specific problems have to be solved by using workarounds. In most cases the applications are tailored specifically to fulfill the needs of the given problem domain. To avoid complexity and to allow for more intuitive structuring of a VE scene graphs like Performer [4] are used.

To allow multiple participants inside a VE, network protocols have to be defined and connection topologies between the different participants have to be chosen. The used communication protocols are closely associated to the underlying topologies. Singhal and Zyda give a detailed introduction into NVE topologies and protocols [5]. A good overview which demonstrates the close connection between multi-user games and NVEs is given in [6].

\section{Conceptual Design}

In the Net'O'Drom game two players compete in a virtual race. They drive their vehicles on a race-track and try to reach the goal before the other player.

\subsection{The Principle of Mutual Dependent Interaction}

It was expected that users with different experience in the area of games and input devices had to interact with each other. Due to that reason an unfair situation during the game would arise which could decrease the gaming experience. In a racing game the more experienced player is able to advance much faster than the inexperienced player, which reduces the aspect of a challenge enormously. To overcome this problem a game mechanism had to be found which keeps the players vehicles in spatial proximity.

\footnotetext{
${ }^{1}$ Formerly known as AVOCADO.
} 
Our approach guarantees this mutual interaction by coupling the players' vehicles with a rubber band mechanism, which keeps the vehicles below a maximal distance. This mechanism generates interesting tactical game situations, whereas the faster player is pulling the slower player along. Both players can apply their acceleration and braking force on the other players' vehicles by pulling the rubber band together. The gaming idea is easy to understand and increases the competition between the players. Even close to the end of the race it is still possible to gain the lead.

\subsection{Technical Implementation of 3D Models}

Due to performance reasons the $3 \mathrm{D}$ models had to be designed using a low amount of polygons. Materials for the objects can be developed with the use of photographic references. A distinction between movable, modular objects and objects of the environment are made. Movable and modular objects (avatars, vehicles and race track tiles) are textured with a diffuse general lighting situation which is defined through the use of skylights and ambient occlusion shadows. This type of lighting generates a strong plasticity and emphasises details. It implements the common light distribution based on the human perception and is perceived as a balanced light by the observers. For the static objects of the VE different daylight situations were defined for the different levels of the game. The objects, background images and architectures are illuminated similar to the movable objects to achieve a level of consistency.

To combine the lighting situation with the lighting-neutral materials of the geometries in common textures a procedure called texture baking is needed. This process guarantees the quality of pre-rendered image quality in a real time application.

The rendering process does not take the camera perspective as a basis for texture calculation, but the UVW-coordinates of the according 3D object. This produces a polygon-based, object projection of light and material attributes on a 3D object per polygon. The result is mapped as a self-illuminated texture with the same UVW coordinates on the given objects. With that mechanism it is possible to achieve an image quality, which is normally known from rendered animations and visualisations.

\section{Game Architecture}

The architecture of the Net'O'Drom NVE consists of a network module, a database module, a physics engine, independent libraries for effects and a highly specific physics engine. A core module combines the independent parts and manages the application. It acts as glue between the individual components and contains the management of events. Graphical output is handled via the scene graph OpenSG, and audio output is generated via the OpenAL audio library. Figure 1 shows the different components and their communication routes. Three different classes of components can be identified which are shown in different shades of 


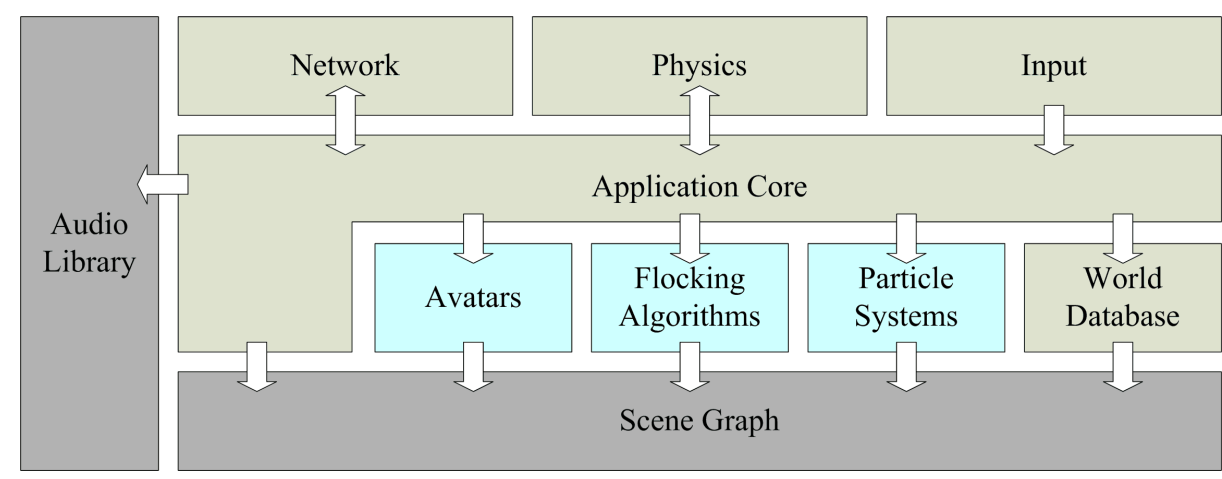

Fig. 1. Overview of the architecture

grey. On the bottom and the left part of the Figure the external libraries are shown. The central part is formed of the actual application core and the modules. In between the application core and the scene graph additional modules which are used to extend the underlying scene graph functionality are shown. These additional modules are implemented as scene graph nodes since they represent graphical effects or non standard geometries which will be explained in detail in Section 4.4, Multi-threading is highly desirable for various components. The input device component and the network component should be independent from the rest of the application and should be polled at certain intervals, to avoid unnecessary waiting times for the application. If physics is used in a VR application it should be performed on a separate processor if available, since physics calculation is normally a computationally intense task.

\subsection{Structuring the VE}

When designing a VE several options arise for the organisation of the scene graph. Ideally nodes should be easy to cull and the structure should be organized and stored in a manner, that the application designer is able to easily change the scene layout without changing the hierarchy of the graph or altering the source code. In our example application the Net'O'Drom racing game proprietary text files define which objects are available in the particular scene and how they are arranged. A VE as structured in the Net'O'Drom application consists of tiles, entities and environments. Additionally each player controls a vehicle to which an avatar and particle systems representing the exhausts of the vehicle are attached.

One environment represents a region of the race track. The tiles and entities represent the visible objects in the world. Tiles are used to structure the race track and can only be arranged on a grid. This approach has been chosen to allow for an intuitive definition inside the configuration files, easy frustum culling and efficient physics calculation. The entities do not have any direct technical purpose and are used to create the atmosphere of the VE. They can be arbitrarily placed 


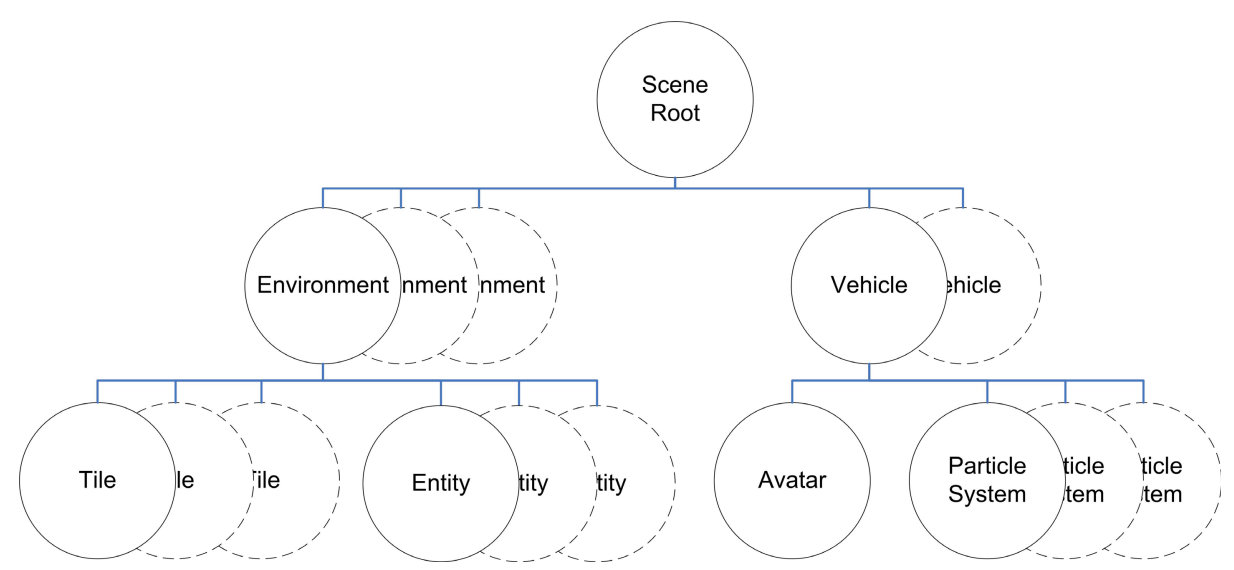

Fig. 2. The scene graph of the Net'O'Drom application

inside an environment. An overview of the scene graph of the example application is given in Figure 2. Organisation of a scene graph should be done in most cases in a way that the different branches of the graph describe certain regions. Ideally the upper nodes in the graph describe a spatially larger region where their child nodes are located inside this region. A common mistake is grouping the different nodes based on their logic, e.g. grouping all tiles or all entities in the scene under one node. Since most scene graph implementations provide optimizations like frustum culling based on the node hierarchy it is recommended to organize the scene based on the spatial position of nodes in the environment.

\subsection{Physics}

A physics engine in the context of computer games and VEs simulates the behaviour of rigid bodies, which comprises the following tasks: rigid body motion, collision detection and response and constrained interaction of rigid bodies. Typically these calculations are performed in 3D space on simplified proxy geometries to reduce the needs of computational power. In our sample application we decided to tailor a highly efficient, problem specific physics engine. The basic idea is to perform as much work as possible in 2D. Therefore a heightmap is calculated based on the surface. In order to perform collision detection in 2D, obstacles and moving objects on the surface are approximated by $2 \mathrm{D}$ primitives.

Heightmap. The heightmap is generated out of a set of geometries which is considered as surface of the area reachable by the vehicle. The height values are obtained by performing intersection tests between the surface geometry and rays going into the direction of gravity. Also the surface normals are kept which are necessary to determine the orientation of objects on the heightmap in 3D. This procedure is time consuming but it needs to be performed only when the surface geometry changes. Queries to the heightmap of the height value or normal for 
a given $(x, y)$ position on the surface are performed by simply interpolating the values of its neighbours.

A limitation to this approach is that each point on the surface can have exactly one height value.

Collision Detection. Collision detection aims to determine the intersection of two objects. Our implementation provides lines and circles as collision primitives. In the simulation the vehicles are represented by circles and the obstacles on the surface are approximated by line sets which describe the $2 \mathrm{D}$ shapes of the obstacles. Every possible collision point on the obstacle's mesh is determined by simulating the vehicle scratching along the obstacle. The result is a line set which describes the collision shape of the obstacle in 3D. These lines are then projected into $2 \mathrm{D}$ space for use in the collision detection algorithm. Since the vehicle is mobile and moves over a non-planar surface its $2 \mathrm{D}$ shape can change over time. To avoid a collision test with changing $2 \mathrm{D}$ shapes our collision detection algorithm considers the orientation of the vehicles. A more detailed description on this approach can be found in [7].

To allow for efficient physics calculation it is always important to know the given application domain. Using a standard approach and working with all of the triangles of the scene will decrease the overall performance enormously. The representation of the scene should be pre-processed and simplified in advance as much as possible to allow for simplified calculations in the physics component.

\subsection{Network}

The network module makes a clear distinction between two types of transmission data, continuous movement data and discrete events. The communication topology is a peer-to-peer (p2p) architecture designed for two users. One client starts the application as a server and the second client has to connect to this server. Once this connection is established, both clients work on their fully replicated copy of data and synchronise the application via messages. For most NVEs we can identify three different types of messages which are used for position update, scene management and management of the network topology.

The first type of messages describes the transformation of the vehicle in the $\mathrm{VE}$ and other physical properties of the vehicles like speed and orientation. To avoid the additional latencies other than the network delay the updates have to be transmitted as fast as possible. Therefore the continuous flow of movement data is distributed via UDP. If the latencies introduced by the network are already to high it is possible to extrapolate the position of the remote users representation, in our case the vehicle with the avatar, by using predictive dead reckoning algorithms.

A second type of messages consists of discrete events which have to be handled in a reliable manner. Events can be used for many purposes, e.g. to trigger animations, to trigger sound effects, or to provide reliable information for the physics engine. These reliable events guarantee a consistent state of an NVE. 
Another message type is used to manage the network topology and is mostly independent from the application. Typical messages which are transmitted could be joining or leaving of a participant of the VE.

An important issue designing network protocols for NVEs lies in the byte order, which differs between standard Intel and AMD CPUs and most of the CPUs used in supercomputers.

\subsection{Graphical Effects}

Graphical effects are useful to increase the degree of immersion and to attract users. Particle systems [8] are often used in safety applications to demonstrate the propagation of smoke or to animate fires and explosions. Flocking [9] algorithms can be used to create a more dynamic and life-like VE.

In general these effects are not necessary for the simulation of the VE, they have to be separated from the actual application layer and should be updated once per frame. Besides their usefulness for enhancing immersion and their ability to create life-like environments these effects have more in common. They can cause significant performance issues when they are used in combination with immersive multi-screen displays. Particle systems emit particles from their core and update each frame the attributes of the set of emitted particles. These updates have to be synchronized between the different displays. Similar problems arise with flocking algorithms, where the boids of the flock have to be synchronised in their position and animation phase. To overcome these probles a collection of boids or a collection of particles has to be implemented as a single node.

Most of these problems can be avoided by using shared memory architectures and synchronizing the memory access where the changes of the nodes come into effect. If the multi-wall display is driven by a cluster system, the distribution of random seeds and the synchronization of these seeds can be used to synchronize particle systems and flocking algorithms.

\section{Conclusions and Future Work}

The actual Net'O'Drom application was demonstrated to the public with great success on an SGI Onyx with an active stereo Curved Screen was used as output display. This system was interconnected to a portable Linux driven PC with a passive stereo powerwall display.

This paper has identified the necessary core parts for a networked VR application. Suggestions on how to design these components were given with the example of Net'O'Drom, a networked racing game. Not only technical aspects like physics or network communication were presented, but also other aspects like graphical design or effects which are used to increase immersion were introduced. Many pitfalls that can be made during the development of NVEs have been shown, and suggestions how to maneuver around these obstacles were given.

Core parts of the Net'O'Drom application are being used in a framework for the design of more generic NVEs - the inVRs framework. An brief overview of 
the inVRs framework is given in 10. Furthermore this application will be used as a demonstration application to show the capabilities of the edutain@grid middleware.

\section{Acknowledgments}

We would like to thank all the people from the Johannes Kepler University and the University of Reading, which helped to implement this application most notably: Helmut Garstenauer, Martin Garstenauer, Adrian Haffegee, Marlene Hochrieser, Franz Keferböck, Stephan Reiter, Christian Wressnegger, and Johannes Zarl. The modelling and design of the VE was done by students of the Kunstuniversität Linz: Wolfgang Hauer, Clemens Mock, Paul Pammesberger, Ivan Petrov, Georg-Friedrich Sochurek, Silke Wiesinger and Wolfgang Wögerer. For additional support for the organization of the public demonstration event we would like to thank Gerhard Funk, Christa Sommerer, and Friedrich Valach.

The work described in this paper is supported in part by the European Union through the IST-034601 project "edutain@grid".

\section{References}

1. Carlsson, C., Hagsand, O.: Dive - a platform for multiuser virtual environments. Computers and Graphics 17 (1993) 663-669

2. Conway, M.J., Pausch, R., Gossweiler, R., Burnette, T.: Alice: A rapid prototyping system for building virtual environments. In: ACM Conference on Human Factors in Computing Systems (CHI '94). Volume 2., Boston, MA, USA, ACM Press (1994) 295-296

3. Tramberend, H.: Avocado: A distributed virtual reality framework. In: IEEE Virtual Reality (VR '99), Houston, TX, USA, IEEE Computer Society (1999) $14-21$

4. Rohlf, J., Helman, J.: Iris performer: A high performance multiprocessing toolkit for real-time3d graphics. In: SIGGRAPH, ACM Press (1994) 381-394

5. Singhal, S.K., Zyda, M.J.: Networked Virtual Environments - Design and Implementation. Addison-Wesley Professional (1999)

6. Smed, J., Kaukoranta, T., Hakonen, H.: A review on networking and multiplayer computer games. Tucs technical report no 454, Turku Centre for Computer Science (2002)

7. Bressler, H., Landertshamer, R., Anthes, C., Volkert, J.: An efficient physics engine for virtual worlds. In: Mediaterra 06. (2006)

8. Reeves, W.T.: Particle systems - a technique for modeling a class of fuzzy objects. ACM Transactions on Graphics 2 (1983) 93-108

9. Reynolds, C.W.: Flocks, herds, and schools: A distributed behavioral model. Computer Graphics 21 (1987) 25-34

10. Anthes, C., Volkert, J.: invrs - a framework for building interactive networked virtual reality systems. In: International Conference on High Performance Computing and Communications (HPCC '06), Munich, Germany, Springer (2006) 894-904 